\title{
COMPARATIVE STUDIES OF SURFACE ROUGHNESS OF THIN EPITAXIAL Si FILMS BY COMPUTER SIMULATIONS AND EXPERIMENTAL X-RAY AND OPTICAL METHODS
}

D. Żymierska, J. Auleytner, J. Domagala, A. Szewczyk

Institute of Physics, Polish Academy of Sciences

Al. Lotników 32/46, 02-668 Warsaw, Poland

AND N. DMITRUK

Institute for Physics of Semiconductors, National Academy of Sciences of Ukraine Prosp. Nauki 45, $252650 \mathrm{Kiev}$, Ukraine

The paper presents investigations of the surface roughness of epitaxial silicon films obtained by chemical vapour deposition with chloric and MOCVD processes. The flat surfaces of films and chemically etched surfaces of substrates were studied by optical methods as well as by X-ray reflectivity at grazing incidence. The computer simulations based on Fresnel theory were compared with the experimental results.

PACS numbers: 61.10.Dp, 68.35.Bs, 78.20.Ci

\section{Introduction}

The surface roughness is a very important factor determining the quality of a crystal surface. When X-rays impinge on the plane surface of a solid, total reflection occurs if the grazing angle, $\Theta_{1}$, has a value less than that of critical angle, $\theta_{c}$, which depends mainly on the electron density of a solid and on the wavelength of incident radiation, $\lambda$. The specular reflectivity, both in the optical and in X-ray range of wavelengths, can be a good tool for surface research on microscopic length scale. Recently, due to synchrotron radiation the sensitivity of the method of grazing incidence reflectivity has been increased very much, and a big progress from the classic paper of Parratt [1] to works of Als-Nielsen et al. [2] and of Dietrich and Haase [3] was made. The aim of the present paper is to determine the surface roughness of the thin silicon films and their substrates by complementary, X-ray and optical techniques and to compare results of both methods as well as to compare these experimental data with results of theoretical calculations. Up to date, the surface roughness of silicon crystals was investigated by several authors, e.g. [4-7]. 


\section{Experimental}

Thin epitaxial silicon films were grown by chemical vapour deposition with chloric (the surface of the film $A$ and of the Si substrate $B$ ) and MOCVD (surfaces $C$ and $D$, respectively) processes. The surfaces $A$ and $C$ are natural, as grown, so called flat. The surfaces of substrates $B$ and $D$ were chemically polished with standard chemical-dynamic etching. The thickness of the film with surface $A$ was equal to $6.0 \mu \mathrm{m}$ and the film with surface $C$ was equal to $5.4 \mu \mathrm{m}$. The geometric parameters of the relief were estimated using M-252 and Dectak-2 profilometers. In particular, the mean-arithmetic deflexion from the mean profile line, $R_{\mathrm{a}}$ was equal to about $5 \mathrm{~nm}$ for surface $A$, to $13 \mathrm{~nm}$ for surface $B$, to $10 \mathrm{~nm}$ for surface $C$ and to $30 \mathrm{~nm}$ for surface $D$.

The roughness of a surface is described by the root-mean-square (RMS) value of surface roughness, $\sigma$, which corresponds to the height of surface irregularities. In the present paper values of $\sigma$ were obtained independently by optical and X-ray techniques. The optical specular reflection spectra were measured at room temperature in the spectral range $\lambda=(4-10.5) \times 10^{2} \mathrm{~nm}$ by MDR-3 spectrometer for different incidence angles with both the suitable attachment and the $\mathrm{Al}$ mirror as etalon.

The X-ray reflectivity at grazing incidence was measured with the $\mathrm{Cu} K_{\alpha_{1}}$ $(\lambda=0.1541 \mathrm{~nm})$ radiation by high resolution Philips MRD equipped with the Bartels monochromator (two channel-cut Ge crystals with two reflections 220 each). In this way, a highly monochromatic X-ray beam is obtained $\left(\Delta \lambda / \lambda=6.9 \times 10^{-5}\right)$ with a low divergence $(\Delta \Theta=12$ arcsec). Additionally, the incident beam was formed with the $50 \mu \mathrm{m}$ vertical slit. The reflected from investigated surface beam was measured with the proportional counter localized behind the narrow $(90 \mu \mathrm{m})$ slit. The size of the detector opening angle $\Delta 2 \Theta=0.03^{\circ}$. The data were collected in classical $\Theta / 2 \Theta$ scan (incidence angle = reflected angle).

\section{Computer simulations}

The theoretical calculations based on Fresnel theory were performed in the way presented in the previous paper [8]. Since the Fresnel equations hold for a smooth surface, the additional damping factor, so-called scattering coefficient $\gamma$ for reflectivity from a rough surface has to be introduced, e.g. [9]. If the Gaussian distribution of the surface roughness is assumed, the reflectivity can be written as

$$
R_{\mathrm{r}}=R_{\mathrm{i}} \exp \left(-4 k_{1}^{2} \sigma^{2} \sin ^{2} \Theta_{1}\right)
$$

where $R_{\mathrm{i}}$ is the reflectivity from a smooth surface and $k_{1}$ is the wave vector of the incident radiation. The coefficient $\gamma$ is equal to the exponent in formula (1). This form of damping is the most widely used but in the case of X-rays it is not sufficient for describing the measured reflectivities. In order to take into account the surface roughness properly the conception of a self-affine surface defined by Mandelbrot [10] was adapted by Sinha et al. [11, 12]. The surface roughness can be characterized by height-height correlation function

$$
C(t)=\sigma^{2} \exp \left[-(t / L)^{2 h}\right]
$$

where $\sigma$ is the RMS value of the surface roughness, $t$ is a distance between two points in $x, y$ plane for which the height-height correlation function is considered, 
$L$ is a roughness correlation length and $h$ is a roughness exponent taking values from 0 for a very jagged surface to 1 for smooth hills and valleys at surface. For $h=1$ the correlation function decays like a Gaussian.

In this case, the scattering coefficient is multiplied by a new additional surface factor given by

$$
S=\left|q_{z}\right|^{-2} \exp \left\{-0.5 \sigma^{2}\left[\left(q_{z}\right)^{2}+\left(q_{z}^{*}\right)^{2}\right]\right\} \int_{0}^{\infty} \mathrm{d} t \cos \left(q_{r} t\right)\left\{\exp \left[\left|q_{z}\right|^{2} \sigma^{2} C(t)\right]-1\right\}
$$

with

$$
q_{r}=0.5 k_{1}\left(\Theta_{1}^{2}-{\Theta^{\prime}}_{2}^{\prime 2}\right) \quad \text { and } \quad q_{z}=k_{1}\left(\Theta_{2}+\widetilde{\Theta}_{2}\right),
$$

where $q$ is the scattering vector.

In the limit $q_{z} \ll 1$, the surface factor $S$ reduces to the Fourier transform of the height-height correlation function.

In the present paper, calculations were performed for silicon surfaces characterized by the index of refraction $n=1-7.573 \times 10^{-6}-\mathrm{i} \cdot 1.755 \times 10^{-7}$ for $\mathrm{Cu}$ $K_{\alpha_{1}}$ radiation. According to the optical measurements, the Gaussian distribution of the surface irregularities was assumed, i.e. $h=1$. The other surface parameters were chosen in a wide range.

\section{Results}

The results of the optical measurements are presented in Fig. 1. According to the formula (1), the value of $\ln \left(R_{\mathrm{r}}\right)$, where $R_{\mathrm{r}}$ is the specular reflection coefficient, is linear versus $\lambda^{-2}$, where $\lambda$ is the wavelength of incident radiation. A slope of this line depends on the square of RMS value of the surface roughness. For chemically polished surfaces of the silicon substrates used for deposition of films both with the chloric (surface $B$ ) and the MOCVD (surface $D$ ) processes straight lines were observed. In Fig. 1 the dependence of $\ln \left(R^{\prime}\right)$ with $R^{\prime}$ being the ratio of the reflection coefficient of a substrate, $R_{\text {rel }}$, to that of a flat surface of film, $R_{\mathrm{f}}$, for surface $B$ is shown.

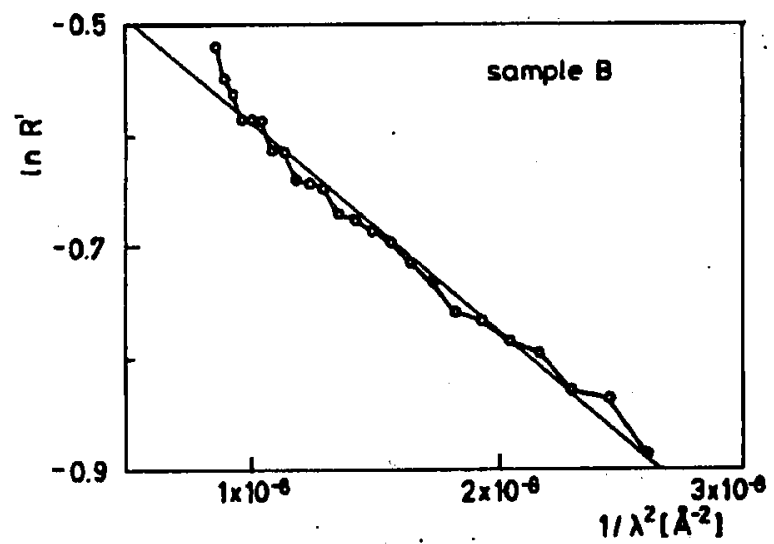

Fig. 1. Value of $\ln R^{\prime}$ versus $1 / \lambda^{2}$ with $\lambda$ being the wavelength of incident optical radiation. 


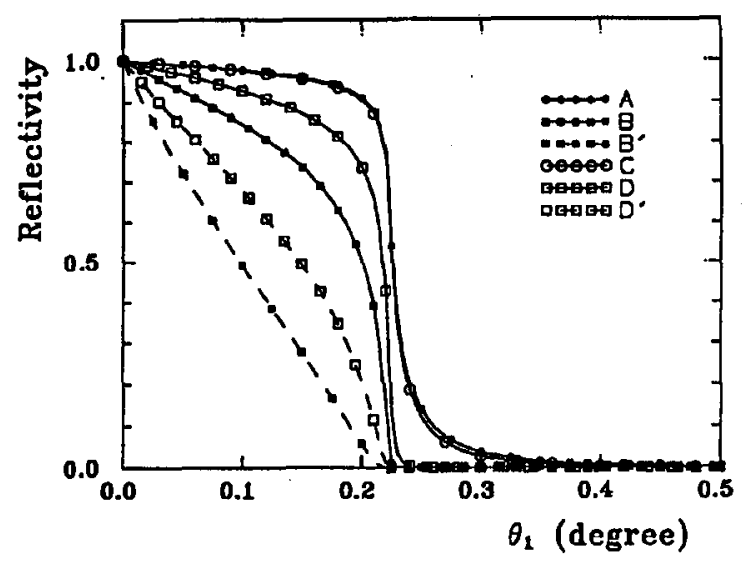

Fig. 2. The fitting of calculated reflectivity curve (full line) to experimental grazing incidence X-ray reflectivity curve (full circles) for surface $C$. The assumed surface parameters are $\sigma=1.2 \mathrm{~nm}, L=1300 \mathrm{~nm}$ and $h=1$.

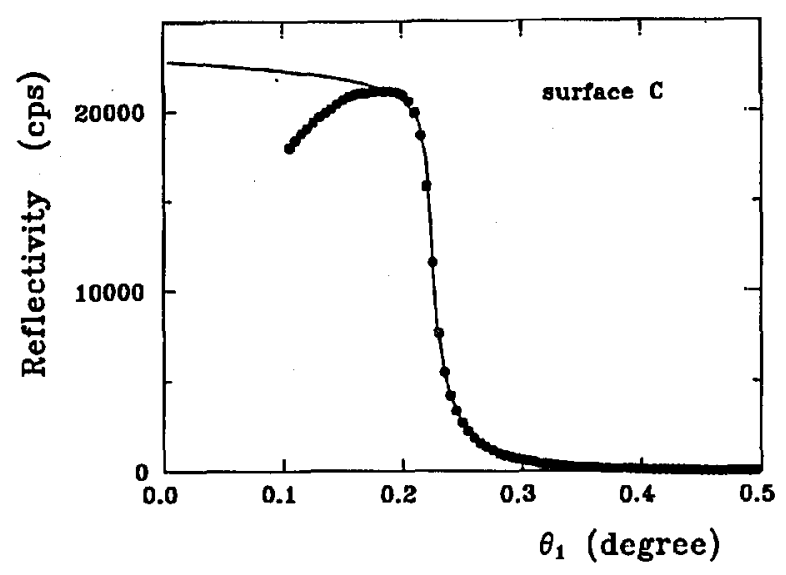

Fig. 3. Simulated grazing incidence X-ray reflectivity curves from $\mathrm{Si}$ surfaces with $\mathrm{Cu} K_{\alpha_{1}}$ radiation. The assumed surface parameters are $h=1, L=1000 \mathrm{~nm}$ and $\sigma$ related to the best fitting of experimental data for each surface. Prime indicates calculation including value $\sigma$ obtained by optical measurements.

The results of X-ray reflectivity measured as a function of the grazing angle $\Theta_{1}$ for surface $C$ is indicated by full circles in Fig. 2. For an interpretation of the experimental data the simulated reflectivity curve was used. By adjusting a theoretical curve (full line) to a measured one (full circles) the values of surface roughness were obtained. In the ideal case of non-absorbing solid with perfectly flat and smooth surface the grazing incidence X-ray reflectivity becomes 1 below critical angle and drops to zero for $\Theta_{1}=1.5 \Theta_{c}$ and this decay is approximately proportional to $\Theta_{1}^{-4}$. For silicon for $\mathrm{Cu} K_{\alpha_{1}}$ radiation the critical angle is equal 
to $0.223^{\circ}$, therefore fitting to the experimental data was performed for angles of incidence smaller than $0.5^{\circ}$. The best fitting was obtained within the angular diapason defined by $0.1805^{\circ}<\Theta_{1}<0.5000^{\circ}$ (the experimental step in incidence angle was equal to $0.0005^{\circ}$ ).

In Fig. 3 the theoretical reflectivity curves are shown. These curves represent the best fitting for each sample, which was obtained for the surface roughness $\sigma=(0.45 \pm 0.08) \mathrm{nm}$ for surface $A$ (full circles), $\sigma=(11.2 \pm 2.8) \mathrm{nm}$ for surface $B$ (full squares), $\sigma=(1.2 \pm 0.3) \mathrm{nm}$ for surface $C$ (open circles) and $\sigma=(6.7 \pm 1.2) \mathrm{nm}$ for surface $D$ (open squares). The circles relate to flat film surfaces and squares to chemically polished substrate surfaces. Full characters designate chloric process of sample deposition and open characters - process with MOCVD. The dashed lines and prime indicate the computer simulation performed for the data obtained by optical measurements: $\sigma=34.2 \mathrm{~nm}$ for surface $B$ and $\sigma=25.0 \mathrm{~nm}$ for surface $D$.

\section{Conclusions}

The aim of our paper was the determination of the surface roughness of the different surfaces of the epitaxial silicon films and the silicon substrates, i.e., the flat surfaces and the chemically etched surfaces, by investigation of the specular reflectivity in two different ranges of wavelength of incident radiation, optical and $\mathrm{X}$-ray ones. The optical measurements were carried out in the spectral range $\lambda=(4-10.5) \times 10^{2} \mathrm{~nm}$ and $\mathrm{X}$-ray measurements with $\mathrm{Cu} K_{\alpha_{1}}$ radiation $(\lambda=$ $0.1541 \mathrm{~nm}$ ). The range of surface roughness which can be examined depends on the wavelength of used radiation. The optical measurements relate to the macro-profile of the surface and X-ray ones to the detailed micro-irregularities, therefore the optical and X-ray techniques are complementary.

A comparison of the theoretical results with experimentally observed X-ray reflectivity curves was also discussed. The experimental intensities at very small angles from $0^{\circ}$ to $0.1110^{\circ}$ were reduced because the investigated surfaces were finite and did not sweep up the whole incident beam. It caused some difficulties in comparison of calculated curves with experimental data. It was estimated that the fitting of the simulated reflectivities to the measured X-ray data can be done with precision of $25-30 \%$.

\section{Acknowledgments}

This study was partly supported by the Committee for Scientific Research (grants Nos. 2PO3B 02109 and 2238892 03). The high resolution Philips MRD diffractometer was founded by the Foundation for Polish Science within the programme SEZAM 94.

\section{References}

[1] L.G. Parratt, Phys. Rev. 95, 359 (1954).

[2] J. Als-Nielsen, D. Jacquemain, K. Kjaer, F. Leveiller, M. Lahav, L. Leiserowitz, Phys. Rep. 246, 251 (1994).

[3] S. Dietrich, A. Haase, Phys. Rep. 260, 1 (1995).

[4] N. Bernhard, E. Burkel, G. Gompper, H. Metzger, J. Peisl, H. Wagner, G. Wallner, Z. Phys. B 69, 303 (1987). 
[5] A. Bensaid, G. Patrat, M. Brunel, F. de Bergevin, R. Herino, Solid State Commun. 79, 923 (1991).

[6] D.K. Bowen, B.K. Tanner, Nanotechnology 4, 175 (1993).

[7] O. Sakata, A.Y. Nikulin, H. Hashizume, Jpn. J. Appl. Phys. 32, L616 (1993).

[8] D. Żymierska, Acta Phys. Pol. A 89, 347 (1996).

[9] P. Beckmann, A. Spizzichino, The Scattering of Electromagnetic Waves from Rough Surfaces, Pergamon Press, Oxford 1963.

[10] B.B. Mandelbrot, The Fractal Geometry of Nature, Freeman, New York 1982.

[11] S.K. Sinha, E.B. Sirota, S. Garoff, H.B. Stanley, Phys. Rev. B 38, 2297 (1988).

[12] S.K. Sinha, Acta Phys. Pol. A 89, 219 (1996). 